\title{
REVIEW
}

\section{Recommendations for peer-to-peer support for NICU parents}

\author{
SL Hall ${ }^{1}$, DJ Ryan ${ }^{2}, J_{\text {Beatty }}{ }^{3}$ and L Grubbs ${ }^{4}$
}

Peer-to-peer support provided by 'veteran' neonatal intensive care unit (NICU) parents to those with current NICU babies is a legitimate and unique form of support that can complement or supplement, but not replace, services provided by professional NICU staff. Peer support can be delivered through hospital- or community-based programs that offer one-to-one in-person or telephone matches, or support groups that meet in-person or via the Internet. Issues in program development, volunteer training and program operation are discussed. Recommendations for offering peer support to all NICU parents as an integral component of family-centered care and comprehensive family support are presented.

Journal of Perinatology (2015) 35, S9-S13; doi:10.1038/jp.2015.143

\section{INTRODUCTION}

Parents who experience a complicated pregnancy or the birth of a baby born prematurely, with congenital anomalies or an illness, face an unknown situation with their baby's hospitalization. The highly specialized care their baby may require leaves families struggling as they cope with parenting from a distance, not feeling like parents and hesitating to become involved. ${ }^{1}$ This new experience leaves parents with a sense of powerlessness, feeling uninformed and intimidated because of their lack of familiarity with the neonatal intensive care unit (NICU) environment. ${ }^{1}$ Families have a vital role in ensuring the health and well-being of their NICU babies, and emotional, social and developmental support of both babies and families are integral components of neonatal health care. ${ }^{2}$ Parents of NICU babies have the opportunity to be better prepared if they have the emotional support and aid of other parents who have been through a similar experience.

Parents with infants in NICUs are well known to be at increased risk for postpartum depression, ${ }^{3-5}$ post-traumatic stress disorder ${ }^{6,7}$ and anxiety. ${ }^{3,5}$ Each of these conditions can disrupt parent-infant bonding, ${ }^{4,8}$ leading to adverse childhood outcomes including worse cognitive, developmental and behavioral functioning. ${ }^{3,9}$ Psychosocial support is critical in mitigating the risk factors for developing these conditions. Although professional mental health staff such as social workers, and to a lesser degree psychologists, may be available to deliver services to NICU parents, not every family may need or want formal support. However, parents may actually experience increased stress from their usual informal support networks because their friends and family may not fully comprehend the NICU experience, ${ }^{10,11}$ and friends and family may also be grieving along with the parents. ${ }^{12}$ NICU parents may also be physically isolated from their traditional support networks, ${ }^{13}$ increasing the potential benefit for receiving peer support services while in the NICU.

Peer-to-peer support ('peer support') is a well-established modality for improving outcomes in people with a wide range of risk factors and diagnoses. ${ }^{14,15}$ In a NICU setting, peer support and parent mentoring are provided by volunteer parents who have had a similar experience ('veteran parents') and who have received training; programs can either be hospital-based or be community-based. The rationale for peer support is that it offers a shared experience in which one NICU parent can best understand what another is going through. ${ }^{10,12,16-19}$ This shared experience allows for acceptance without judgment and provides a foundation of respect between parents. ${ }^{16,18}$ Peer support programs in NICUs can serve to foster a feeling of safety and comfort among parents; ${ }^{20}$ parents can share their fears, get validation for their feelings and gain perspective. ${ }^{18}$ Such programs can also serve as a platform for parents' questions and provide encouragement for parents to become advocates for their babies and themselves. ${ }^{1}$

There is now a growing body of evidence of the benefits that peer support provides to parents of NICU infants and special needs children. Parents who receive peer support have been found to have increased confidence ${ }^{18,21,22}$ and well-being, ${ }^{18}$ problem-solving capacity ${ }^{18,22}$ and adaptive coping, ${ }^{10}$ perception of social support, ${ }^{16}$ self-esteem ${ }^{23}$ and acceptance of their situation. ${ }^{22}$ Further, parents feel more empowered ${ }^{18}$ and interact with, nurture and care for their infants to a greater degree ${ }^{23,24}$ during more frequent visits to the hospital, ${ }^{25}$ leading to a shorter length of stay for their infants. ${ }^{26}$ Parental stress and anxiety, as well as depression, are all reduced. ${ }^{16,21,23,27,28}$ Peer support therefore offers a 'legitimate ${ }^{\prime 23}$ and 'unique form of assistance that is not typically met by the formal service system ${ }^{\prime 22}$ and one that cannot come from any other source. ${ }^{19}$ The support provided by volunteer mentors from externally developed peer support organizations should never duplicate or replace formal/professional support provided to parents by NICU staff; ${ }^{22,29,30}$ it can, however, supplement and complement internal professional services offered by the hospital. ${ }^{20}$ Pediatricians and other health-care professionals should facilitate and encourage peer support, $^{18,22,29,30}$ as recommended by the American Academy of Pediatrics in $2012 .^{2}$ As a core principle of family-centered neonatal care, it should be an integral component of every NICU family support program.

\footnotetext{
${ }^{1}$ Division of Neonatology, St John's Regional Medical Center, Oxnard, CA, USA; ${ }^{2}$ School of Nursing, Elmira College, Elmira, NY, USA; ${ }^{3}$ Program Director, Hand to Hold, Austin, TX, USA and ${ }^{4}$ Founder and President, NICU Helping Hands, Fort Worth, TX, USA. Correspondence: Dr SL Hall, Division of Neonatology, St John's Regional Medical Center, 1600 N. Rose Avenue, Oxnard, CA 93030, USA. 


\section{TYPES OF PEER-TO-PEER SUPPORT}

The support for families in the NICU can be as unique as the families themselves. All types of support can be provided by mentors from peer support organizations, whether they are hospital-based or community-based; support provided may be emotional, informational or tangible, which might include concrete physical, financial or material assistance. ${ }^{27}$

A variety of models for providing peer support exist, and many parent support organizations offer blended models in which parents can utilize more than one-and sometimes even all-modalities. Frequency of contact and duration of contact during the NICU stay and beyond to home are dependent on each program and on individual participants.

In-person support or telephone support

In this model, veteran parents are closely matched on as many dimensions as possible (language, culture, baby's diagnosis, family makeup and so on $)^{10,18}$ with current NICU parents to serve as their mentors or 'buddies'. Through the relationship that parent mentors establish with new NICU parents, they can normalize the parents' situation ${ }^{18,31}$ and help them accept it by modeling a positive attitude. ${ }^{22}$ Mentors can reduce parents' isolation, ${ }^{18,29}$ sometimes even serving as substitute family and friends. ${ }^{10}$ This may be particularly important to low-income parents who may not have well-functioning support networks of their own. ${ }^{22}$ The support is provided in non-medical language that is easier for parents to understand than that provided by the health-care team. ${ }^{22}$ Mentors can also give parents practical advice, help them resolve day-to-day problems ${ }^{12}$ and help them access other services. ${ }^{22}$ Although parents may feel that they need to 'hold it together' when dealing with the medical team so that they appear capable, having a support person not affiliated with the medical team allows parents to let their guard down and express their true emotions. ${ }^{16}$ Within the frequently changing NICU landscape, a parent mentor can provide a parent with continuity of care throughout their NICU experience and even beyond. ${ }^{29}$

One potential shortcoming of this model of support is that it may be difficult to ensure that all matches between mentors and parents are good ones, especially when parents are non-English speaking and/or from different cultures. ${ }^{10,18}$ Communication styles, parenting styles, outlook on life and parents' vision of the future may differ in a particular match. ${ }^{18}$ Matches may also become problematic if the mentor parent has not experienced what current parent is going through, ${ }^{10}$ especially if the mentor's child is doing better than the matched parent's child. ${ }^{18}$ For any of these reasons, matches may need to be changed.

Providing peer support by telephone, instead of in-person, may offer several advantages to stressed NICU parents who may not have time or the ability to meet with their peers and friends. ${ }^{27}$ Connections between mentors and parents can occur more spontaneously and with greater flexibility. ${ }^{16,32}$ Mentors' aroundthe-clock availability, when parents need them, gives parents a feeling of dependability. ${ }^{18}$ In addition, telephone support is private and non-stigmatizing. ${ }^{32}$ It can be effective for low-income mothers with diverse ethnic backgrounds, ${ }^{16}$ and is easily accessible to all regardless of socioeconomic status or geographic location. ${ }^{33}$ Another important benefit is that parents do not need child care in order to make use of telephone support. ${ }^{16}$

Finding appropriate matches for telephone support relationships may be difficult, just as with in-person matches. Parents may ultimately not find their match to be helpful, ${ }^{16}$ and either party can lose their match's phone numbers that can end the relationship or mentor parents may be negligent in following through. Alternatively, parents with babies in the NICU may simply be too busy to utilize phone support. ${ }^{18}$ Among the studies on the value of in-person or telephone peer support for NICU parents, mothers were the primary recipients of support services; there is minimal research on how fathers might utilize and respond to peer support in the NICU. This should be an area for future research.

Parent support groups

Parent support groups can be run by a veteran parent in collaboration with a NICU staff medical (nurse or doctor) or mental health (social worker or psychologist) professional; or groups can be run by parents only or professionals only. However, groups have greater stability and longevity if they are jointly run by a parent and a NICU staff person. ${ }^{24}$ Parents may prefer to have counseling professionals over medical professionals as group leaders. ${ }^{34}$ Groups generally have goals of providing information and education, as well as support to parents. ${ }^{29,31}$ Through participation in a group, parents learn how to interact with their babies and get more involved with their care; subsequently, parents gain greater confidence in parenting. ${ }^{25,29,31}$ Group participation further leads parents to visit their baby more often in the NICU and to show greater interest in their baby's development after discharge. ${ }^{25}$ In the group, parents also get coaching on how to interact and collaborate with baby's medical team, ${ }^{29}$ and learn how to communicate more effectively about their baby's and their own needs. ${ }^{31}$ Parents get insight into their feelings, feel less isolated ${ }^{29}$ and have the opportunity to interact with others who are in a similar situation. ${ }^{31}$ Another benefit that may be seen when NICUs offer support groups is that the NICU staff can become more responsive to the needs of families through hearing about parents' concerns. ${ }^{31}$

However, not all parents find value in group support; ${ }^{34}$ participating in a group may actually increase some parents stress. $^{29,30}$ They may not feel comfortable sharing in a group setting in front of strangers, ${ }^{30}$ and may not want to hear other parents' stories about the complications their babies encountered. ${ }^{17}$

It can be challenging to establish and maintain group attendance, ${ }^{34}$ especially in smaller NICUs. Groups may be poorly attended by new mothers who are depressed, ${ }^{35}$ and parents whose babies have shorter stays may not feel the need for group support. ${ }^{36}$ Other barriers to parents' use of support groups include their lack of child care and/or transportation ${ }^{34}$ and the inconvenience of evening meetings for some parents. ${ }^{17}$ For all these reasons, outreach from either NICU staff or veteran parents is required to encourage parental involvement. Parents may be more likely to attend groups if the groups are identified as being primarily educational in nature, rather than 'support groups'.

If professionals run support groups, then it is important that they allow participants to learn from each other by giving up their role as expert. Changes in hospital staffing, or lack of commitment of group leaders to continue the group in spite of obstacles, may lead to a group's dissolution. ${ }^{24}$

\section{Internet support groups}

Parents of NICU babies are increasingly turning to Internet support groups and web pages, with the profusion of parent support organizations that now have an online presence. These sites may be monitored by veteran parents and/or by health-care providers including social workers or nurses, ${ }^{37}$ although there is potential for the presence of professionals to inhibit parental exchanges. ${ }^{27}$

A key benefit to Internet support is that parents can access support on sites tailored to their needs anytime and from anywhere, ${ }^{27}$ which may be particularly beneficial to parents who reside in rural areas, ${ }^{38}$ and to young single mothers and those who are socially isolated. ${ }^{27}$ Variables that can inhibit face-to-face communication, such as differences in gender, age and social class, are reduced when parents interact on websites. ${ }^{39}$ Parents have access to both information and support, and if they are socially sensitive, they can anonymously read others' posts. The 
collective knowledge of the group is available to all, regardless of their participation level. ${ }^{27}$ Information can also be imparted through live chats online; these can be moderated by professionals, increasing their value. ${ }^{37}$ Many parents find Internet support sites to be especially helpful after NICU discharge.

There are several potential drawbacks to parents' use of Internet support sites. Emotionally charged or negative interactions, including abrasive or non-supportive posts, may occur as a result of the reduced social presence people have online. ${ }^{27}$ Information that is shared between parents may be misleading or unreliable, especially if the site lacks professional oversight to ensure that discussions between participants are medically sound. Veteran parents need to make it clear that they are not medical providers and that parents should consult their child's own doctor for medical issues.

\section{BARRIERS TO PARENTS' ACCESSING PEER SUPPORT}

The size and budget of a NICU most often determine what level of formal support can be provided to families as well as whether the NICU can embrace a peer support program and whether it is hospital- or community-based. Even when peer support programs are offered to NICU parents, many families still encounter barriers to accessing them. Each family's needs may vary, making it difficult for a peer support program to provide a best fit for all. Redundancy of professional and peer support services may lead to underutilization of the latter, ${ }^{17}$ and inadequate cultural awareness on the part of staff as well as their limited availability to do outreach may also reduce families' access to peer support services.

Some NICU families may not feel justified in using support services. This may be the case if they are experiencing a shorter NICU stay, if they have a full-term baby in the NICU or feel their baby is not having major health issues or if they do not feel the need for support until they have been in the NICU for a longer period of time. Parents often do not want to leave their baby's bedside to access support; time constraints may be another limitation. Transportation issues, especially for mothers who have undergone a cesarean delivery, or financial difficulties can further impede parents' ability to access hospital-based peer support services. In addition, parent's lack of awareness of available services may limit their use. ${ }^{17}$

\section{ISSUES IN PROGRAM DEVELOPMENT}

Veteran parent support is grounded in the experiential knowledge of parents who have effectively coped with parenting a NICU baby over time and at home. ${ }^{23}$ Veteran parents offer shared common experiences and know the stress associated with a baby's diagnosis, have weathered the many transitional times and have sifted through services so that they have practical knowledge of available resources. ${ }^{19}$ The goals of peer support programs should include the following: to provide informal psychosocial support to all families whose babies have been admitted to the NICU; to increase both parents' coping and parenting skills so they gain the confidence they need to feel that they are part of the NICU team and the competence to care for their baby; to provide information to families about hospital and community resources for their baby and family and to assist families in making the transition from hospital to home. ${ }^{20}$ An ideal peer support program would provide services to parents beginning in the antepartum period, when appropriate, and continuing through their baby's NICU hospitalization and after discharge. Services would also be extended to siblings and grandparents, and to bereaved parents.

To implement a peer support program within a hospital setting, the need must be identified, collaborative commitment from hospital and administrative personnel received, a coordinator secured, and veteran parent mentors recruited and trained. Veteran NICU parents who did not receive peer support themselves and/or hospital personnel who are aware of the benefits of a peer support program may identify the need. The choice of a coordinator could either be a hospital employee (usually a nurse or social worker) or a veteran NICU parent from the hospital. Guidelines related to utilization of volunteers within the patient care setting need to be established and agreed upon before implementation of the program. Recruitment and training of volunteer parent mentors should be done by a professional such as a social worker and/or a parent leader as an ongoing process. Diversity of the NICU population should be reflected in the diversity of the parent mentors to allow for greater capacity in matching NICU parents with mentors. ${ }^{40}$ The NICU experience of parent mentors should have been at least 1 year before starting mentorship, ${ }^{23}$ and any unresolved issues with their own NICU experience need to be addressed before serving as a parent mentor. ${ }^{12,20}$ Parent mentors working in a hospital setting usually need to be screened and approved by the hospital's regular volunteer program as well, to provide them with official volunteer standing in the hospital. ${ }^{20}$ Once a program is begun, ongoing collaboration between hospital and parent mentors is necessary to maintain a successful program, ${ }^{20}$ and institutions need to prioritize their commitment to the program so that even if key personnel leave the NICU environment, the parent support program will continue. For more detailed information about how to start a volunteer peer support program, see the document 'Starting a Parent Support Group' (www.support4nicuparents.org).

\section{VOLUNTEER TRAINING}

Programs utilizing veteran parents to provide peer support to NICU families, regardless of whether support is provided in-person, by phone or Internet, should offer a comprehensive training program designed to equip parent mentors with the skills needed. Content of training may include:

\section{Defining the roles of a parent mentor:}

a. To actively and empathetically listen to and encourage NICU parents without trying to solve their problems or give them advice; ${ }^{19,20,40}$

b. To assist parents in identifying and utilizing their current support systems;

c. To offer emotional and informational support;

d. To encourage advocacy for their baby;

e. To empower parents to step into their parental role as an essential member of their NICU baby's care team; and

f. To identify when to ask for help and where help is available for parents that mentors are supporting. ${ }^{20}$

\section{Defining expectations of a parent mentor:}

a. To maintain confidentiality: the manner in which each hospital handles the sharing of patient information should be communicated;

b. To treat all parents with respect, regardless of their cultural, racial and ethnic backgrounds, socioeconomic status and religion; and

c. To make a time commitment for the program.

\section{Development of essential skill set:}

a. Basic communication and reflective listening skills;

b. Understanding the expected and normal emotional responses of NICU parents, and responses that indicate parents might be at risk for more serious mental health issues;

c. Understanding the dynamics of grief and loss; ${ }^{30}$ and

d. Knowledge of hospital and community resources. 
Parent mentor training should be offered to groups of 8 to 12 parents whenever practical, allowing for a group that is small enough to result in intimacy but large enough to stimulate conversation and interaction. ${ }^{20}$ Demonstrations and observations, role-play, practice interviews and videotaped interviews ${ }^{10}$ can be utilized as training methods. Mentors should be encouraged to reflect on their own NICU experience and consider how it may affect their provision of support to NICU parents. ${ }^{23}$ Ongoing supervision of peer mentors is recommended to ensure that both group facilitators and individual mentors are supported and provided guidance when needed.

\section{ISSUES IN PROGRAM OPERATION}

NICU parents should be offered peer support soon after admission, usually within the first 3 days, ${ }^{20,23}$ and many NICUs offer a program brochure at the time of the baby's admission. Parents may also request support at any time, and hospital or volunteer staff may personally reach out to parents. Parameters for matching volunteer veteran parents with current parents include the baby's gestational age and diagnosis, parents' age, family size, structure and lifestyle, education and income level, language and ethnic background and geographic location. ${ }^{16}$ The more similarities between the NICU experiences of matched parents, the faster and easier it is for a support parent to establish rapport with the new parent. ${ }^{20}$ Matches on the dimensions of culture and language are important determinants of satisfaction for non-English speaking mothers. ${ }^{10}$ Current NICU mothers who have a history of substance abuse, child welfare issues, domestic violence or psychiatric illnesses probably will be matched with veteran mothers who do not share those same experiences. However, trained parents can establish supportive relationships with parents of different backgrounds because of the commonality of the NICU experience, a fact that sets parents apart from NICU professionals. ${ }^{20}$ All parent matches should be followed up to determine their suitability, and the opportunity to change mentor/ buddies should be available. ${ }^{18}$

Multiple challenges may be encountered when starting a peer mentoring program. NICU staff may believe that their care is already family-centered; or staff may be overly focused on technology and medical care of the baby, concerned that a parent-run program will adversely impact their work, or skeptical that parents can meet other parents' needs. In addition to overcoming the NICU staff's cultural resistance and desire to maintain the status quo, other potential barriers in the NICU environment include lack of space, limited time and resources to support volunteers, staff concerns about the safety needs of the baby ${ }^{41}$ and privacy concerns of the family. To ensure the quality of programs offered, continuous quality improvement processes should be implemented.

\section{SUMMARY}

Peer-to-peer support has a legitimate role in the spectrum of providing psychosocial support to NICU parents, ${ }^{23}$ and should be institutionalized as an integral component of family-centered neonatal care that is available to all families with infants in the NICU. ${ }^{29}$

\section{RECOMMENDATIONS}

1. Every parent of every baby admitted to a Special Care Nursery or NICU should be offered peer support from a 'veteran' NICU parent mentor during their baby's hospital stay. Ideally, this opportunity would be a part of a NICU's comprehensive family support program., 40,32

2. While in-person peer support during the baby's hospital stay may be viewed as best practice, peer support can be offered to parents in a variety of different ways including email or phone support, group support or through an online community support site for NICU families.

3. Peer support in any form is best started in the antepartum period when appropriate (as when mothers are hospitalized during the antepartum period), continued through the baby's NICU stay, as well as after baby's discharge from the NICU. Having a consistent peer mentor throughout a NICU parent's journey may be desirable.

4. Parents whose baby expires should be offered the opportunity to be paired with a bereavement mentor who has also experienced a neonatal loss.

5. Peer support is one element of a comprehensive family support program. Other elements should include:

a. An institutional and administrative champion for peer and family support;

b. A facilitator/coordinator who could be:

i. A hospital employee (psychologist, social worker, nurse, pastoral care, physician),

ii. A trained parent and/or family support specialist,

iii. A former NICU parent or volunteer from one of the many non-profit peer-to-peer parent support organizations across the country;

c. A menu of types of support available (individual and/or group in-person support, email, phone and online support);

d. Parent education classes and written materials, as well as social activities;

e. Staff education to promote understanding of NICU families' psychosocial needs, family-centered care practices and methods of communicating and providing support (see 'Recommendations for enhancing psychosocial support of NICU parents through staff education and support', this issue).

6. Any family support program that utilizes peer volunteers should provide training to the volunteers to ensure that they are capable of carrying out the peer support role in a responsible and culturally sensitive way that is fully compliant with both Health Insurance Portability and Accountability Act (HIPPA) regulations and hospital policies. ${ }^{33,36}$

7. In NICUs without the resources to develop a local and comprehensive family support program of their own, parents should be referred to regional or national parent support organizations that have been evaluated by their NICU staff. Resources for parent support can be found at websites of the following organizations: the National Perinatal Association's Family Advocacy Network (www.nationalperinatal.org/parent supportgroups), the Preemie Parent Alliance (http://preemiepar entalliance.weebly.com/\#/), the March of Dimes (www.share yourstory.org), the Canadian Premature Babies Foundation (www.cpbf-fbpc.org) and the European Foundation for the Care of Newborn Infants (www.efcni.org).

8. Peer support organizations working with NICU families should consider offering support services to members of the baby's family including siblings, grandparents ${ }^{12,29}$ and others, as needed and desired.

\section{CONFLICT OF INTEREST}

SL Hall has a consulting agreement with the Wellness Network, but this organization had no input or editing rights to the content included in the recommendations. The remaining authors declare no conflicts of interest.

\section{ACKNOWLEDGEMENTS}

Many thanks to other participants from the National Perinatal Association Workgroup on Psychosocial Support of NICU Parents for their contributions to this work, 
including Tawna Burton, Becky Hatfield, Kristy Love, Heather McKinnis and Jennifer Schum. This article has been supported by grants and contributions from: the Wellness Network, Prolacta Bioscience, Division of Neonatology at Loma Linda University School of Medicine, NICU Parent Support at Mercy Hospital in St Louis, MO, Hand to Hold, Preemie Parent Alliance, Zoe Rose Memorial Foundation, the Rosemary Kennedy Trust and Eden's Garden.

\section{REFERENCES}

1 Bracht M, O'Leary L, Lee S, O'Brien K. Implementing family-integrated care in the NICU: a parent education and support program. Adv Neonatal Care 2013; 13(2): 115-126.

2 American Academy of Pediatrics, Committee on Hospital Care, Institute for Patient- and Family-Centered Care. Patient- and family-centered care and the pediatrician's role. Pediatrics 2012; 129: 394-404.

3 Singer L, Salvator A, Guo S, Collin M, Lilien L, Baley J. Maternal psychological distress and parenting stress after the birth of a very low-birth-weight infant. JAMA 1999; 281: 799-805.

4 Miles S, Holditch-Davis D, Schwartz T, Scher M. Depressive symptoms in mothers of prematurely born infants. J Dev Behav Pediatr 2007; 28: 36-44.

5 Rogers C, Kidokoro H, Wallendorf M, Inder T. Identifying mothers of very preterm infants at-risk for postpartum depression and anxiety before discharge. J Perinatol 2013; 33: 171-176.

6 Callahan J, Borja S, Hynan M. Modification of the Perinatal PTSD Questionnaire to enhance clinical utility. J Perinatol 2006; 26: 533-539.

7 DeMier R, Hynan M, Harris H, Manniello R. Perinatal stressors as predictors of symptoms of posttraumatic stress in mothers of infants at high risk. J Perinatol 1996; 16: 276-280.

8 Bernard-Bonnin A, Psychosocial Paediatrics Committee of Canadian Society of Pediatrics, Canadian Paediatric Society. Maternal depression and child development. Pediatr Child Health 2004; 9: 575-583.

9 Huhtala M, Korja R, Lehtonen L, Haataha L, Lapinleimu P, Rautava P et al. Parental psychological well-being and behavioral outcome of very low birth weight infants at 3 years. Pediatrics 2012; 129: e937-e944.

10 Ardal F, Sulman J, Fuller-Thomson E. Support like a walking stick: parent-buddy matching for language and culture in the NICU. Neonatal Netw 2011; 30: 89-98.

11 Feldman-Reichman S, Miller A, Gordon R, Hendricks-Munoz K. Stress appraisal and coping in mothers of NICU infants. Child Health Care 1998; 29: 279-293.

12 Kerr S, Mclntosh J. Coping when a child has a disability: exploring the impact of parent-to-parent support. Child Care Health Dev 2000; 26: 309-322.

13 Bennett D, Slade $\mathrm{P}$. Infants born at risk: consequences for maternal postpartum adjustment. Br J Med Psychol 1991; 64: 159-172.

14 Humphreys K, Wing S, McCarty D, Chappel J, Gallant L, Haberle B et al. Self-help organizations for alcohol and drug problems: toward evidence-based practice and policy. Psychiatry Res 2004; 26: 151-158.

15 Dixon L, Lucksted A, Stewart B, Burland J, Brown C, Postrado L et al. Outcomes of the peer-taught 12-week family-to-family education program for severe mental illness. Acta Psychiatr Scand 2004; 109: 207-215.

16 Preyde M, Ardal F. Effectiveness of a parent 'buddy' program for mothers of very preterm infants in a neonatal intensive care unit. CMAJ 2003; 168: 969-973.

17 Nottage SL. Parents' use of nonmedical support services in the Neonatal Intensive Care Unit. Issues Compr Pediatr Nurs 2005; 28: 257-273.

18 Ainbinder J, Blanchard L, Singer G, Sullivan M, Powers L, Marquis J et al. A qualitative study of parent to parent support for parents of children with special needs. J Pediatr Psychol 1998; 23: 99-109.

19 Santelli B, Turnbull A, Higgins C. Parent to parent support and health care. Pediatr Nurs 1997; 23: 303-306.

20 Jarrett M. Parent partners: a parent-to-parent support program in the NICU. Part II: program implementation. Pediatr Nurs 1996; 22: 142-149.

21 Cooper L, Gooding J, Gallagher J, Sternesky L, Ledsky R, Berns S. Impact of a family-centered care initiative on NICU care, staff and families. J Perinatol 2007; 27: S32-S37.
22 Singer G, Marquis J, Powers L, Blanchard L, Divenere N, Santelli B et al. A multi-site evaluation of parent to parent programs for parents of children with disabilities. J Early Interv 1999; 22: 217-229.

23 Roman L, Lindsay J, Boger R, DeWys M, Beaumont E, Jones A et al. Parent-toparent support initiated in the neonatal intensive care unit. Res Nurs Health 1995; 18: 385-394.

24 Boukydis C. Support services and peer support for parents of at-risk infants: an international perspective. Child Health Care 2000; 29: 129-145.

25 Minde K, Shosenberg N, Marton P, Thompson J, Ripley J, Burns S. Self-help groups in a premature nursery--a controlled evaluation. J Pediatr 1980; 96: 933-940.

26 Nearing G, Salas A, Granado-Villar D, Chandler B, Soliz A. Psychosocial parental support programs and short-term clinical outcomes in extremely low-birthweight infants. J Matern Fetal Neonatal Med 2012; 25: 89-93.

27 Dunham P, Hurshman A, Litwin E, Gusella J, Ellsworth C, Dodd P. Computermediated social support: single young mothers as a model system. Am J Community Psychol 1998; 26: 281-306.

28 Ireys $\mathrm{H}$, Chernoff R, DeVet K, Kim Y. Maternal outcomes of a randomized controlled trial of a community-based support program for families of children with chronic illnesses. Arch Pediatr Adolesc Med 2001; 155: 771-777.

29 Hurst I. One size does not fit all: parent's evaluation of a support program in a newborn intensive care nursery. J Perinat Neonatal Nurs 2006; 20: $252-261$.

30 Lindsay J, Roman L, DeWys M, Eager M, Levick J, Quinn M. Creative caring in the NICU: parent-to-parent support. Neonatal Netw 1993; 12: 37-44.

31 Bracht M, Ardal F, Bot A. Initiation and maintenance of a hospital-based parent group for parents of premature infants: key factors for success. Neonatal Netw 1998; 17(3): 33-37.

32 Thome $M$, Alder B. A telephone intervention to reduce fatigue and symptom distress in mothers with difficult infants in the community. J Adv Nurs 1999; 29(1): 128-137.

33 Galinsky M, Schopler J, Abell M. Connecting group members through telephone and computer groups. Health Soc Work 1997; 22(3): 181-188.

34 Smith K, Gabard D, Dale D, Drucker A. Parental opinions about attending parent support groups. Child Health Care 1994; 23(2): 127-136.

35 Dennis C-L, Hodnett E, Kenton L, Weston J, Zupancic J, Stewart D et al. Effect of peer support on prevention of postnatal depression among high risk women: multisite randomised controlled trial. BMJ 2009; 338: 280-284.

36 Dobbins N, Bohlig C, Sutphen J. Partners in growth: implementing familycentered changes in the neonatal intensive care unit. Child Health Care 1994; 23 (2): 115-126.

37 Scharer K. Internet social support for parents: the state of science. J Child Adolesc Psychiatr Nurs 2005; 18: 26-35.

38 McNamara D. Health-oriented telecommunication: a community resource. Nurs Manage 1994; 25(12): 40-41.

39 Kiesler S, Siegel J, McGuire T. Social psychological aspects of computer mediated communication. Am Psychol 1984; 39(10): 1123-1134.

40 Santelli B, Parent to Parent USA. Guidance for Recruiting and Training Support Parents. Parent to Parent USA Guidance for Recruiting And Training Support Parents, 2010. Available from: http://www.p2pusa.org/p2pusa/P2P\%20Documents/Guidance \%20for\%20Recruiting\%20and\%20Training\%20Support\%20Parents-1.doc.pdf (date last accessed 24 March 2015).

41 Jarrett M. Parent partners: a parent-to-parent support program in the NICU. Part 1: program development. Pediatr Nurs 1996; 22(1): 60-63.

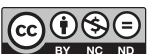

This work is licensed under a Creative Commons AttributionNonCommercial-NoDerivs 4.0 International License. The images or other third party material in this article are included in the article's Creative Commons license, unless indicated otherwise in the credit line; if the material is not included under the Creative Commons license, users will need to obtain permission from the license holder to reproduce the material. To view a copy of this license, visit http:// creativecommons.org/licenses/by-nc-nd/4.0/ 